\title{
Novel Bi-directional Relaying Schemes in a Wireless Multi-Hop Network
}

\author{
Shengyao Jin*, Honglin $\mathrm{Hu}^{*}$, François Gagnon ${ }^{\dagger}$ and Olivier Munger ${ }^{\dagger}$ \\ * Shanghai Research Center for Wireless Communications \\ Shanghai, P. R. China \\ Email: shengyao.jin@shrcwc.org,hlhu@ieee.org \\ †École de technologie supérieure \\ Montreal, Canada \\ Email: Francois.Gagnon@etsmtl.ca,olivier.munger@lacime.etsmtl.ca
}

\begin{abstract}
The exchange of independent information between two nodes in a wireless network can be viewed as a twoway traffic flow. Such information exchange can be efficiently performed by exploiting relaying technique in a two-way channel. In this paper, we introduce two novel relaying techniques for Bidirectional Amplification of the Throughput (BAT) relaying in wireless multi-hop networks, termed as Equalized Detect-andForward (EDF) and Pre-equalized Amplify-and-Forward (PAF) BAT relaying schemes respectively. It is shown that the EDF BAT relaying scheme can decrease the complexity of the relay node by equalizing at the relay node, and the PAF BAT relaying scheme can reduce the signaling overhead by pre-equalizing at the transmitter. Furthermore, we analyze the achievable throughput of these relaying schemes, considering the impact of the location of the relay node.
\end{abstract}

Index Terms-Relay, Network Coding, Bi-directional, Preequalization.

\section{INTRODUCTION}

In recent years, relaying becomes a hot research area [1]. Predictably, for the next generation wireless system, relay will play an important role by providing broader coverage to a large number of users e.g. commuters with trains. Moreover, relay could be used in an ad hoc solution for applications which do not require large wireless resources. However, it is inevitable that the communication phases will increase due to the feature of the half duplex in the relaying system.

Two way communication channel was first studied by Shannon [2], where he contributed an inner bound and an outer bound on the capacity region. Recently, many researches are studying the two-way communication for relay channel, i.e., two end nodes want to exchange messages whereas a third node helps both nodes in the communication process, which is based on the framework of network coding in order to save network resources [3]. For one way communication, although several transmission strategies are well known: Decode-andForward (DF) [4], [5], compress-and-forward [4], [5] or Amplify-and-Forward (AF) [6], the related study of the two way communication for the relay channel is not sufficient. In [7], it is shown that, the spectral efficiency of the two way relaying is significantly increased compared to that of the one way communication. Thereby, how to apply corresponding transmission techniques into the two way communication is

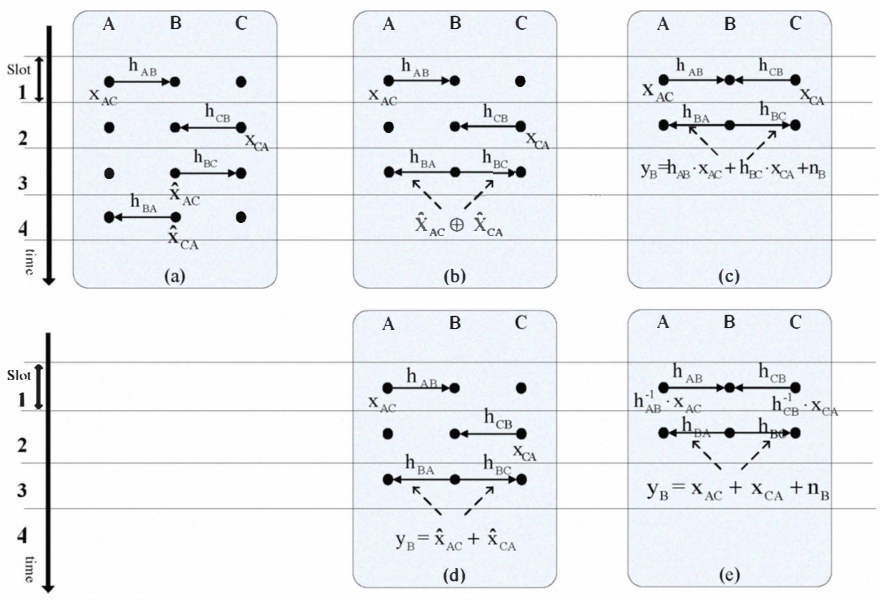

Fig. 1: (a)Conventional bi-directional relaying

(b) Conventional DF BAT relaying

(c) Conventional AF BAT relaying

(d) EDF BAT relaying

(e) PAF BAT relaying

demanding and challenging.

In [8] and [9], the authors have presented three conventional bi-directional relaying schemes, termed as conventional bidirectional relaying scheme, conventional DF Bi-directional Amplification of Throughput (BAT) relaying scheme and conventional $A F B A T$ relaying scheme, respectively. For the conventional bi-directional relaying scheme, as depicted in Fig.1 (a), it takes four phases to exchange information between two end nodes. Comparably, for the conventional DF BAT relaying scheme, the relay node applies a network coding operation and broadcasts the composite data, hence, the performance of the conventional DF BAT relaying scheme can be improved due to the less transmission time; nevertheless requiring decoding the data into bit level. For the conventional AF BAT relaying scheme, only two phases are needed, however, the receiver must know the channel information between the relay and the source nodes to detect the data, thus the signaling overhead increases. 
In order to mitigate the above-mentioned weaknesses while keeping optimal performance, we propose two novel relaying schemes which increase the achievable throughput over the conventional relaying schemes. In the Equalized Detect-andForward (EDF) BAT relaying scheme, as depicted in Fig. 1 (d), we apply equalization technique at the relay node to decrease the complexity of relay. Comparably, the Pre-equalized Amplified-and-Forward (PAF) BAT relaying scheme, as shown in Fig.1 (e), can reduce the signaling overhead compared to the conventional AF BAT relaying scheme by using preequalization technique at the transmitter.

The rest of this paper is organized as follows. Section II gives the system model and introduces the three conventional schemes. Section III describes the two proposed bi-directional relaying schemes and discusses the impact of the location of the relay node. Numerical results are then provided in Section IV to further evaluate the advantages of the proposed relaying schemes. Finally, Section V concludes this paper.

\section{System Model And Conventional Relaying SCHEMES}

To illustrate the concept of the bi-directional traffic in a wireless network, we consider the system model depicted in Fig.1, where the end node $A$ and $C$ act as both sources and receivers, and $B$ operates as an intermediate relay node. In this paper, we focus on an Orthogonal Frequency Division Multiplexing (OFDM) based Time Division Duplex (TDD) system.

We suppose that node $A$ has a stream of packets $x_{A C}$ and node $C$ has another stream of packets $x_{A C}$, and they aim to exchange their information. Traditionally, without the presence of the relay node $B$, communication between node $A$ and $C$ is performed in two steps: first the node $A$ transmits its packets to $C$ followed by the similar transmission from node $C$ to $A$. When introducing an intermediate relay node $B$, the most straightforward extension to the notion of downlink and uplink is to use four orthogonal time slots, depicted in Fig. 1 (a) [10].

Assuming that $x_{A C}$ and $x_{C A}$ are the complex baseband in symbol level and their expected values are $E\left\{x_{A C}\right\}=$ $E\left\{x_{C A}\right\}=0$ and $E\left\{\left|x_{A C}\right|^{2}\right\}=E\left\{\left|x_{C A}\right|^{2}\right\}=1$. Without loss of generality, we can consider that the average transmitted energy of source and relay is normalized, i.e., $P_{A}=P_{B}=$ $P_{C}=1$.

In the conventional $D F B A T$ relaying scheme, after decoding the received data, the relay node $B$ applies a network coding operation and broadcasts the packets $x_{B}=\hat{x}_{A C} \oplus \hat{x}_{C A}$, where $\oplus$ denotes the bitwise XOR operation. Since the node $A$ already has the knowledge of $x_{A C}$, it extracts the required packets $x_{C A}$ through $\hat{x}_{C A}=x_{B} \oplus x_{A C}$. Similarly, the node $\hat{x}_{A C}=x_{B} \oplus x_{C A} C$ extracts . The relaying method in Fig. 1 (b) requires only 3 time slots to transfer the packets $x_{A C}$ and $x_{C A}$ [8]. We can see that the conventional DF BAT relaying scheme is limited within the bit level operation.

Another efficient BAT relaying scheme is proposed by Petar Popovski [9] as in Fig. 1 (c), namely conventional $A F B A T$ relaying scheme. In contrast to the conventional $D F B A T$ relaying scheme, both node $A$ and $C$ transmit $x_{A C}$ and $x_{C A}$ to the relay node $B$ simultaneously during $1^{\text {st }}$ time slot. After that, the relay node $B$ amplifies superposition of the data packets from node $A$ and $C$, and broadcasts them to the two end nodes simply. It can be noticed that the channel state information (CSI) between the relay and the source nodes are required at the destiny node, which implies a huge and impractical signaling overhead.

\section{Proposed Bi-directional Relaying Schemes}

In this section, we will introduce two proposed BAT relaying schemes, i.e., EDF BAT relaying scheme depicted in Fig. 1 (d) and the $P A F$ BAT relaying scheme illustrated in Fig. 1 (e). Then, we will briefly discuss the impact of the location of the relay node.

\section{A. EDF BAT relaying scheme}

In the $E D F B A T$ relaying scheme, we apply equalization at the relay node $B$ in stead of decoding in the conventional $D F$ $B A T$ relaying scheme as depicted in Fig. 1 (b).

During the $1^{\text {st }}$ time slot, the node $A$ transmits its packets to the relay node $B$, and the received symbols at the relay node $B$ is

$$
y_{E, B 1}=h_{A B} x_{A C}+n_{B},
$$

where the subscript $E$ denote the $E D F B A T$ relaying scheme. In addition, $h_{a b}$ denotes the channel fading coefficient between the node $a$ and $b$. For example, $h_{A B}$ is the channel fading coefficient between the node $A$ and $B$. Furthermore, $n_{B}$ is a complex valued Additive White Gaussian Noise (AWGN) with variance $\sigma_{B}^{2}$ at the receiver of the relay node $B$.

Then we perform equalization at the relay node $B$ with the CSI between the node $A$ and the relay node $B$. Moreover, and assume that $h_{A B}^{-1} h_{A B}=1$, which means the channel estimation is perfect and equalization can compensate the signal distortion completely. Thus, the transmitted signal from the node $A$ can be estimated by the hard-decision in symbol level as

$$
\begin{aligned}
\hat{x}_{A C} & =h_{A B}^{-1} y_{E, B 1} \\
& =h_{A B}^{-1}\left(h_{A B} x_{A C}+n_{B}\right) \\
& =x_{A C}+h_{A B}^{-1} n_{B} .
\end{aligned}
$$

Similarly during the 2 nd time slot, the received signal is equalized and the transmitted signal from the node $C$ can be estimated by the hard-decision in symbol level as

$$
\begin{aligned}
\hat{x}_{C A} & =h_{C B}^{-1} y_{E, B 2} \\
& =h_{C B}^{-1}\left(h_{C B} x_{C A}+n_{B}\right) \\
& =x_{C A}+h_{C B}^{-1} n_{B} .
\end{aligned}
$$

After the hard-decision, the symbols from both the node $A$ and $C$ are summed as

$$
y_{E, B}=\hat{x}_{A C}+\hat{x}_{C A} .
$$

During the $3^{r d}$ time slot, the relay node $B$ amplifies $y_{E, B}$ with a normalized factor $\beta_{E}$ and broadcasts it to both the node 
$A$ and $C$. Then the received signal at the node $A$ can be written as

$$
\begin{aligned}
y_{E, A} & =\beta_{E} h_{B A} y_{E, B}+n_{A} \\
& =\beta_{E} h_{B A}\left(\hat{x}_{A C}+\hat{x}_{C A}\right)+n_{A} .
\end{aligned}
$$

The average transmitted signal energy over one symbol period at the relay node $B$ is the same as that of the node $A$, i.e., we have

$$
\begin{aligned}
\beta_{E} & =\sqrt{\frac{1}{E\left\{\left|\hat{x}_{A C}\right|^{2}\right\}+E\left\{\left|\hat{x}_{C A}\right|^{2}\right\}}} \\
& =\sqrt{\frac{1}{2}} .
\end{aligned}
$$

With the knowledge of $h_{B A}, x_{A C}$ and $\beta_{E}$, the estimation of the $x_{C A}$ can be expressed as

$$
\hat{x}_{C A}=\beta_{E}^{-1} h_{B A}^{-1} r_{E, A},
$$

where

$$
\begin{aligned}
r_{E, A} & =y_{E, A}-\beta_{E} h_{B A} x_{A C} \\
& =\beta_{E} h_{B A} \hat{x}_{A C}+\beta_{E} h_{B A} \hat{x}_{C A}+n_{A}-\beta_{E} h_{B A} x_{A C} \\
& =\beta_{E} h_{B A} \hat{x}_{C A}+n_{A}+\beta_{E} h_{B A}\left(\hat{x}_{A C}-x_{A C}\right) .
\end{aligned}
$$

Similarly, the estimation of the $x_{A C}$ can be given as

$$
\hat{x}_{A C}=\beta_{E}^{-1} \hat{h}_{B C}^{-1} r_{E, C},
$$

where

$$
r_{E, C}=\beta_{E} h_{B C} \hat{x}_{A C}+n_{C}+\beta_{E} h_{B C}\left(\hat{x}_{C A}-x_{C A}\right) .
$$

\section{B. PAF BAT relaying scheme}

Pre-equalization technique has been proposed to simplify the symbol detection at the receiver especially for a TDD based transmission due to the channel reciprocity [11]. As depicted in Fig. 1 (e), the PAF BAT relaying scheme uses preequalization to avoid the impractical signaling overhead as in the conventional $A F B A T$ relaying scheme.

During the $1^{s t}$ time slot, node $A$ and $C$ transmit $h_{A B}^{-1} x_{A C}$ and $h_{C B}^{-1} x_{C A}$ respectively to the relay node $B$ simultaneously with pre-equalization. Then, the received data at the relay node $B$ can be written as

$$
\begin{aligned}
y_{P, B} & =h_{A B} h_{A B}^{-1} x_{A C}+h_{C B} h_{C B}^{-1} x_{C A}+n_{B} \\
& =x_{A C}+x_{C A}+n_{B},
\end{aligned}
$$

where the subscript $P$ denote the $P A F$ BAT relaying scheme.

During the $2^{\text {nd }}$ time slot, the relay node $B$ amplifies with a normalized factor $\beta_{P}$ expressed as

$$
\begin{aligned}
\beta_{P} & =\sqrt{\frac{1}{E\left\{\left|x_{A C}\right|^{2}\right\}+E\left\{\left|x_{C A}\right|^{2}\right\}+\sigma_{B}^{2}}} \\
& =\sqrt{\frac{1}{2+\sigma_{B}^{2}}} .
\end{aligned}
$$

Then, the relay node $B$ broadcasts the amplified signal to both the node $A$ and $C$, where the average transmitted signal energy over one symbol period at the relay node $B$ is the same as that at the node $A$. Now, the received signal at the node $A$ is

$$
\begin{aligned}
y_{P, A}= & \beta_{P} h_{B A} y_{P, B}+n_{A} \\
= & \beta_{P} h_{B A} x_{A C}+\beta_{P} h_{B A} x_{C A}+\beta_{P} h_{B A} n_{B} \\
& +n_{A} .
\end{aligned}
$$

Again, with the knowledge of $h_{B A}, x_{A C}$ and $\beta_{P}, x_{C A}$ can be estimated as

$$
\hat{x}_{C A}=\beta_{P}^{-1} h_{B A}^{-1} r_{P, A},
$$

where

$$
\begin{aligned}
r_{P, A}= & \beta h_{B A} x_{A C}+\beta P h_{B A} x_{C A}+\beta P h_{B A} n_{B}+n_{A} \\
& -\beta P h_{B A} x_{A C} \\
= & \beta P h_{B A} x_{C A}+\beta P h_{B A} n_{B}+n_{A} .
\end{aligned}
$$

Similarly, $x_{A C}$ can be estimated as

$$
\hat{x}_{A C}=\beta_{P}^{-1} h_{B C}^{-1} r_{P, C},
$$

where

$$
r_{P, C}=\beta h_{B C} x_{A}+\beta h_{B C} n_{B}+n_{C} .
$$

It can be noticed that the $P A F B A T$ relaying scheme can estimate the signals from the source node at the destiny node without requiring the knowledge of CSI between the source and the relay node. When the node A receives the signal from the node $C$, it is unnecessary to know the CSI between the relay node $B$ and the node $C$ since we perform the preequalization at the node $A$ earlier. Then the only requirement to detect the signal from $C$ is CSI between the node $A$ and the relay node $\mathrm{B}$. If we do not perform pre-equalization, we have to know the CSI between not only the node $A$ and the relay node $B$ but also the relay node $B$ and the node $C$. Consequently, the signaling overhead will increase significantly. Otherwise the inference between and could not be cancelled.

\section{Impact of the location of the relay node}

To consider the effect of the location of the relay node on the network performance, we follow the model in Fig. 2. Moreover, we assume that the relay node is located on the line connecting the two end nodes and both $A-B$ and $B-C$ are static line-of-sight (LOS) links without fast fading. Supposing that the amplitude squares of the channel coefficients are exponentially distributed with $\lambda_{A B}, \lambda_{B A}, \lambda_{C B}, \lambda_{B C}, \lambda_{A C}$, and $\lambda_{C A}$. The parameters for the exponential distributions capture the effect of pathloss across the corresponding link. In addition, we normalize the distance between the two end nodes. Denote the distance from the node $A$ to the relay node $B$ as $d$, then the distance between the relay node $B$ and the node $C$ is $1-d$, where $0<d<1$. Then the overall network channel state becomes a 3-section independent exponential random variables with $\lambda_{A C}=\lambda_{C A}=1, \lambda_{C B}=\lambda_{B C}=\frac{1}{(1-d)^{\alpha}}$ 


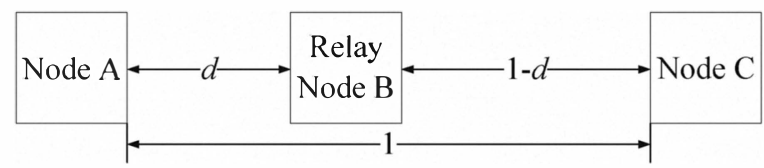

Fig. 2: The model of Bi-directional relaying

and $\lambda_{C B}=\lambda_{B C}=\frac{1}{(d)^{\alpha}}$, respectively, where $\alpha$ is the pathloss exponent.

As in [10], the EDF BAT relaying and the PAF BAT relaying schemes can be modeled by using an appropriate Markov chain, thus derive the achievable throughput. The packet error rate (PER) on link $A-B, B-A, C-B, B-C, A-C$ and $C-A$ are denoted by $p_{e 1}, p_{e 2}, p_{e 3}, p_{e 4}, p_{e 5}$, and $p_{e 6}$ respectively. Moreover, the duration of a time slot for one packet transmission is denoted by $T_{S}$. Thus, the normalized data rate for a packet of $N$ bits becomes $R_{S}=\frac{N}{T_{S}}$. Then, we can conclude that the throughput of the conventional bidirectional relaying, the conventional $D F B A T$ relaying, the conventional $A F B A T$ relaying, the $E D F B A T$ relaying, and the $P A F B A T$ relaying schemes are respectively

$$
\begin{gathered}
R_{C o n}=\frac{R_{s}\left(\frac{\left(1-p_{e 1}\right)\left(1-p_{e 3}\right)}{2-p_{e 1}-p_{e 3}}+\frac{\left(1-p_{e 2}\right)\left(1-p_{e 4}\right)}{2-p_{e 2}-p_{e 4}}\right)}{2}, \\
R_{C D F}=\frac{R_{s}\left(2-p_{e 2}-p_{e 4}\right)}{3}, \\
R_{C A F}=\frac{R_{s}\left(2-p_{e 5}-p_{e 6}\right)}{2},
\end{gathered}
$$

$$
\begin{gathered}
R_{E D F} \\
=\frac{R_{s}\left(1-p_{e 1}\right)\left(1-p_{e 3}\right)(2}{\left(3-2 p_{e 1}-2 p_{e 3}-p_{e 2}-p_{e 4}+p_{e 2} p\right.} \\
R_{P A F}=\frac{R_{s}\left(2-p_{e 5}-p_{e 6}\right)}{2} .
\end{gathered}
$$

To compare the impact of the different locations of the relay node $B$, we introduce the following notations for the per-symbol signal to noise ratio (SNR), which are respectively given by

$$
\begin{gathered}
S N R_{C o n, A B}=S N R_{C o n, B A}=\frac{\lambda_{A B}}{\sigma^{2}}=\frac{1}{\sigma^{2} d^{\alpha}} \\
S N R_{C o n, C B}=S N R_{C o n, B C}=\frac{\lambda_{C B}}{\sigma^{2}}=\frac{1}{\sigma^{2}(1-d)^{\alpha}}, \\
S N R_{C D F, A B}=S N R_{C D F, B A}=\frac{\lambda_{A B}}{\sigma^{2}}=\frac{1}{\sigma^{2} d^{\alpha}}
\end{gathered}
$$

$$
S N R_{C D F, C B}=S N R_{C D F, B C}=\frac{\lambda_{C B}}{\sigma^{2}}=\frac{1}{\sigma^{2}(1-d)^{\alpha}},
$$

$$
S N R_{E D F, A B}=\frac{\lambda_{A B}}{\sigma^{2}}=\frac{1}{\sigma^{2} d^{\alpha}},
$$

$$
\begin{gathered}
S N R_{E D F, C B}=\frac{\lambda_{C B}}{\sigma^{2}}=\frac{1}{\sigma^{2}(1-d)^{\alpha}}, \\
S N R_{E D F, B A}=\frac{\beta^{2} \lambda_{B A}}{\sigma^{2}}=\frac{1}{2 \sigma^{2} d^{\alpha}},
\end{gathered}
$$

$$
S N R_{E D F, B C}=\frac{\beta^{2} \lambda_{B C}}{\sigma^{2}}=\frac{1}{2 \sigma^{2}(1-d)^{\alpha}},
$$

$$
\begin{aligned}
S N R_{P A F, A C} & =S N R_{C A F, A C} \\
& =\frac{S N R_{C D F, A B} S N R_{C D F, B C}}{2 S N R_{C D F, B C}+S N R_{C D F, A B}+1}(3,1) \\
S N R_{P A F, C A} & =S N R_{C A F, C A} \\
& =\frac{S N R_{C D F, C B} S N R_{C D F, B A}}{2 S N R_{C D F, B A}+S N R_{C D F, C B}+1}(32)
\end{aligned}
$$

Here we suppose QPSK modulation during the transmission and let $P_{b}(S N R)$ be the bit error probability in the AWGN channel. For packets consisting of $N$ bits, the packet error rates can be expressed as

$$
p_{e}=1-\left(1-P_{b}(S N R)\right)^{N},
$$

where

$$
P_{b}(S N R)=Q\left(\sqrt{\frac{2 E_{b}}{N_{0}}}\right)=Q\left(\sqrt{\frac{E_{S}}{N_{0}}}\right) .
$$

(21) By using the $p_{e}$, we could calculate the achievable throughput of the different relaying schemes.

\section{Numerical Results}

First, simulations are performed to evaluate the normalized throughput of the proposed schemes over the conventional schemes in various locations of the relay node. Note that the normalized throughput of the conventional $A F$ BAT relaying scheme is the same as that of the $P A F B A T$ relaying scheme due to the AWGN channel. Therefore, in our simulation, we only show the results for the $P A F B A T$ relaying scheme. Fig. 3 and Fig. 4 show the normalized throughput $\frac{R_{C} o n}{R_{S}}, \frac{R_{C} D F}{R_{S}}$, $\frac{R_{P} D F}{R_{S}}$, and $\frac{R_{P} A F}{R_{S}}$ under different SNR scenarios in AWGN channel. For Fig. 3, we have $\sigma_{A}^{2}=\sigma_{B}^{2}=\sigma_{C}^{2}=0.2$ whereas for Fig. 4, there is $\sigma_{A}^{2}=\sigma_{B}^{2}=\sigma_{C}^{2}=0.15$. In both figures, the packet size $N$ is 100 bits and the pathloss exponent alpha is 2 . From Fig. 3 and Fig. 4, we can conclude that the conventional $D F B A T$ relaying scheme always outperforms the $E D F B A T$ relaying scheme. However, the performance of the $E D F B A T$ 


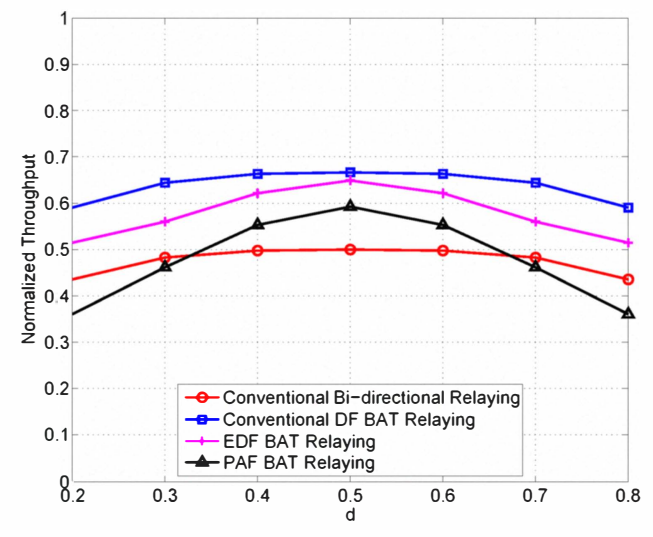

Fig. 3: Normalized throughput versus the distance between $\mathrm{A}-\mathrm{B}, \sigma_{A}^{2}=\sigma_{B}^{2}=\sigma_{C}^{2}=0.2$

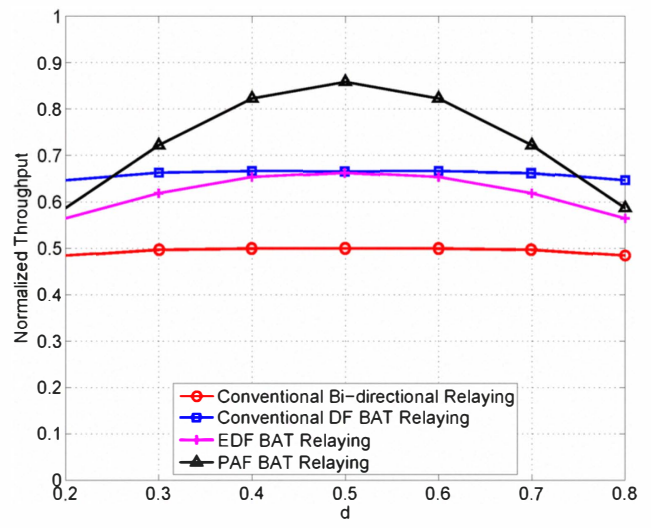

Fig. 4: Normalized throughput versus the distance between A-B, $\sigma_{A}^{2}=\sigma_{B}^{2}=\sigma_{C}^{2}=0.15$

relaying scheme is closer to that of the conventional $D F B A T$ relaying scheme when the relay node $B$ is located in the middle of two end nodes. Furthermore, Fig. 4 demonstrates that the PAF BAT relaying scheme outperforms the rest of the schemes when the noise variance at the receiver is low. Since all the relaying schemes have the best performance when the relay node $B$ keeps the same distance to the two end nodes, we will evaluate and discuss the performance of the different relaying schemes under such a scenario in the following part.

We do not consider about the factor of pathloss in the following simulation, i.e., we assume relay is located in the middle of two end nodes. Moreover, we assume that the two end nodes can not communicate directly and the simulation parameters are summarized in Table I.

Fig. 5 and Fig. 6 illustrate the spectral efficiency vs. SNR of the conventional and the proposed schemes taking QPSK and 16QAM modulation respectively. From the figures we could see that the spectral efficiency can be considerably impacted by the pre-equalization. We assume that the target SNR is around $15 \mathrm{~dB}$ and use it as a reference for the performance comparison. In the low SNR region, it can be noticed that the
TABLE I: Simulation Parameters

\begin{tabular}{|c|c|}
\hline Modulation & QPSK,16QAM \\
\hline Channel coding & $1 / 3$ Turbo code \\
\hline Block length & 450 Symbols \\
\hline Channel model & Rayleigh \\
\hline Detector & MMSE \\
\hline Channel estimator & Ideal \\
\hline Location of the relay node & Middle of two end node \\
\hline
\end{tabular}

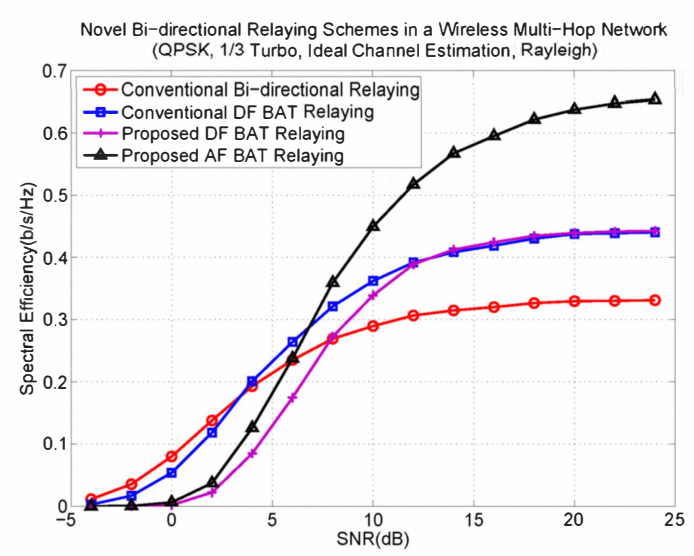

Fig. 5: Spectral Efficiency vs. SNR for Bi-directional Relaying Schemes

conventional bi-directional relaying scheme is the best due to the noise enhancement by the pre-equalization in the $P A F B A T$ relaying scheme. For the $E D F B A T$ relaying scheme without pre-equalization, interference cancellation induces some performance loss due to the serious error propagation in low SNR. However, the latency delay of the conventional bi-directional relaying scheme is the highest among all the schemes.

Furthermore, Fig. 5 and Fig. 6 show that the conventional $D F B A T$ relaying scheme outperforms the $E D F B A T$ relaying scheme in the target $\mathrm{SNR}$, i.e., when $\mathrm{SNR}=15 \mathrm{~dB}$. However, we should note that the $E D F B A T$ relaying scheme does not need the decoding and XOR processes as those in the conventional $D F$ BAT relaying scheme, thus greatly simplify the signal processing at the relay node $B$.

In high SNR region, we could see that the $P A F$ BAT relaying scheme outperforms the rest of four schemes remarkably due to the least time slots, and the performance of the $E D F B A T$ relaying scheme is approaching to that of the conventional $D F B A T$ relaying scheme. Here, we do not simulate the performance of the conventional $A F B A T$ relaying scheme which requires huge CSI signaling, because this scheme is not practical in a real system.

\section{CONClusion}

In this paper, we illustrate two novel bi-directional relaying schemes and analyze the impact of the location of the relay node in a wireless multi-hop network. The EDF BAT relaying scheme can achieve similar performance as the conventional $D F B A T$ relaying scheme whereas the complexity at the 


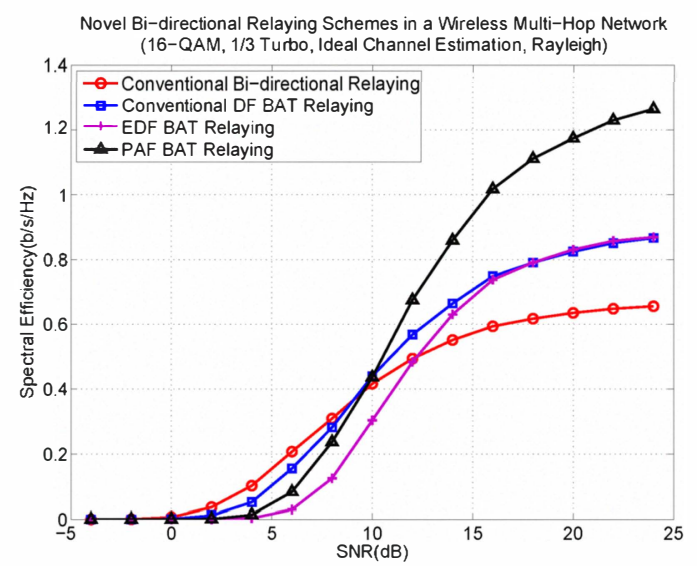

Fig. 6: Spectral Efficiency vs. SNR for Bi-directional Relaying Schemes

relay node can be remarkably reduced. Comparably, the $P A F$ $B A T$ relaying scheme can decrease the required time slots for data transmission. Moreover, the pre-equalization helps to reduce the signaling overhead, although it also slightly increases the complexity at the transmitter side and induces noise enhancement. Finally, it is shown that the middle of the two end nodes is the best location for the relay node in a bi-directional relaying network.

\section{ACKNOWLEDGEMENT}

This work is supported by projects 2008DFA12190, 2008DFA11700, 2008DFA12090, and 2010DFB10570 from MOST of China and 08DZ2200800 from STCSM.

\section{REFERENCES}

[1] A. Sendonaris, "Advanced Techniques for Next-Generation Wireless Systems", Ph.D. Thesis, Rice University, Aug. 1999.

[2] C. E. Shannon, "Two-way communication channels", in Proc. 4th Berkeley Symp. Math. Stat. and Prob, vol. 1, pp. 611-644, 1961.

[3] R. Ahlswede, N. Cai, S.-Y. R. Li, and R. W. Yeung, "Network Information flow", IEEE Trans. Inform. Theory, vol. 46, pp. 1204-1216, Jul. 2000.

[4] T. M. Cover and A. El Gamal, "Capacity Theorems for The Relay Channel", IEEE Trans. Inform. Theory, vol. 25, pp. 572-584, Sept. 1979.

[5] G. Kramer, M. Gastpar, and P. Gupta, "Cooperative Strategies and Capacity Theorems for Relay Networks", IEEE Trans. Inform. Theory, vol. 51, pp. 3037-3063, Sept. 2005.

[6] J. N. Laneman, D. N. Tse, and G. W. Wornell, "Cooperative Diversity in Wireless Networks: Efficient Protocols and Outage Behavior", IEEE Trans. Inform. Theory, vol. 50, pp. 3062-3080, Dec. 2004.

[7] B. Rankov and A. Wittneben, "Spectral Efficient Signaling for Halfduplex Relay Channels", in Proc. Asilomar Conference on Signals, Systems and Computers, Pacific Grove, Nov. 2005.

[8] P. Larsson, N. Johansson and K.-E. Sunell, "Coded Bi-directional Relaying", Proc. IEEE VTC'06 Spring, vol. 2, pp.851-855, 2006.

[9] P. Popovski and H. Yomo, "Bi-directional Amplification of Throughput in a Wireless Multi-Hop Network", in Proc. IEEE VTC'06 Spring, vol. 2, pp. $588-593,2006$

[10] IST-2003-507581 WINNER, "D3.4: Definition and assessment of relay based cellular deployment concepts for future radio scenarios considering 1st protocol characteristics", , Jun. 2005.

[11] K. Witrisal, Y. H. Kim, R. Prasad, and L. P. Lighthart, "Pre-equalization for the Up-link of TDD OFDM Systems", in Proc. IEEE PIMRC '01, vol. 2, pp. 93-98, 2001 